Advances in Intelligent Systems and Computing 923

Ana Maria Madureira Ajith Abraham

Niketa Gandhi

Maria Leonilde Varela Editors
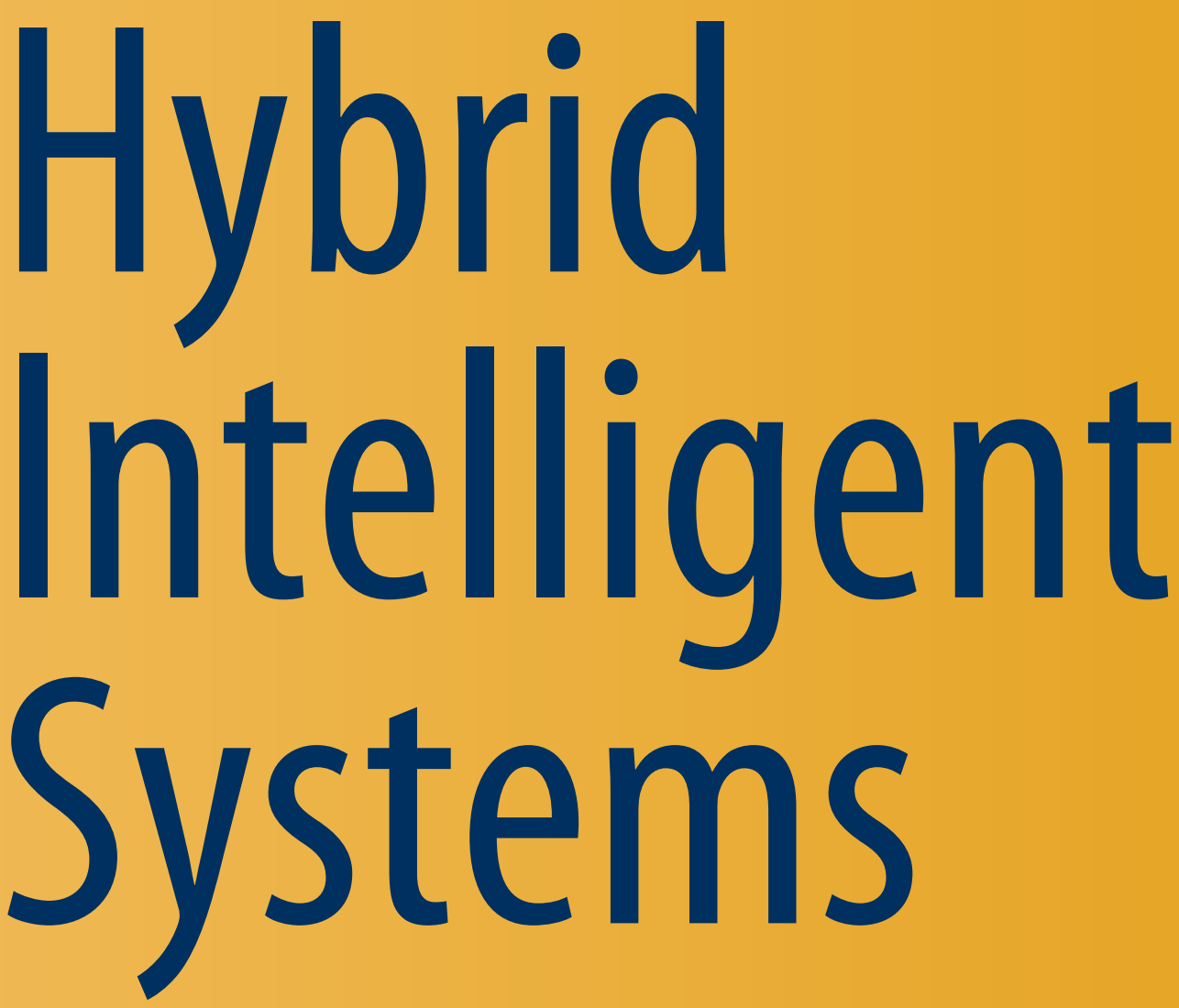

18th International Conference on Hybrid Intelligent Systems (HIS 2018) Held in Porto, Portugal, December 13-15, 2018 


\section{Advances in Intelligent Systems and Computing}

Volume 923

Series editor

Janusz Kacprzyk, Systems Research Institute, Polish Academy of Sciences, Warsaw, Poland 
The series "Advances in Intelligent Systems and Computing" contains publications on theory, applications, and design methods of Intelligent Systems and Intelligent Computing. Virtually all disciplines such as engineering, natural sciences, computer and information science, ICT, economics, business, e-commerce, environment, healthcare, life science are covered. The list of topics spans all the areas of modern intelligent systems and computing such as: computational intelligence, soft computing including neural networks, fuzzy systems, evolutionary computing and the fusion of these paradigms, social intelligence, ambient intelligence, computational neuroscience, artificial life, virtual worlds and society, cognitive science and systems, Perception and Vision, DNA and immune based systems, self-organizing and adaptive systems, e-Learning and teaching, human-centered and human-centric computing, recommender systems, intelligent control, robotics and mechatronics including human-machine teaming, knowledge-based paradigms, learning paradigms, machine ethics, intelligent data analysis, knowledge management, intelligent agents, intelligent decision making and support, intelligent network security, trust management, interactive entertainment, Web intelligence and multimedia.

The publications within "Advances in Intelligent Systems and Computing" are primarily proceedings of important conferences, symposia and congresses. They cover significant recent developments in the field, both of a foundational and applicable character. An important characteristic feature of the series is the short publication time and world-wide distribution. This permits a rapid and broad dissemination of research results.

** Indexing: The books of this series are submitted to ISI Proceedings, EI-Compendex, DBLP, SCOPUS, Google Scholar and Springerlink **

\section{Advisory Editors}

Nikhil R. Pal, Indian Statistical Institute, Kolkata, India

Rafael Bello Perez, Faculty of Mathematics, Physics and Computing, Universidad Central de Las Villas, Santa Clara, Cuba

Emilio S. Corchado, University of Salamanca, Salamanca, Spain

Hani Hagras, Electronic Engineering, University of Essex, Colchester, UK

László T. Kóczy, Department of Automation, Széchenyi István University, Gyor, Hungary

Vladik Kreinovich, Department of Computer Science, University of Texas at El Paso, EL PASO, TX, USA

Chin-Teng Lin, Department of Electrical Engineering, National Chiao Tung University, Hsinchu, Taiwan

Jie Lu, Faculty of Engineering and Information Technology, University of Technology Sydney, Sydney, NSW, Australia

Patricia Melin, Graduate Program of Computer Science, Tijuana Institute of Technology, Tijuana, Mexico

Nadia Nedjah, Department of Electronics Engineering, University of Rio de Janeiro, Rio de Janeiro, Brazil

Ngoc Thanh Nguyen, Faculty of Computer Science and Management, Wrocław University of Technology, Wrocław, Poland

Jun Wang, Department of Mechanical and Automation Engineering, The Chinese University of Hong Kong, Shatin, Hong Kong

More information about this series at http://www.springer.com/series/11156 
Ana Maria Madureira - Ajith Abraham .

Niketa Gandhi · Maria Leonilde Varela

Editors

\section{Hybrid Intelligent Systems}

18th International Conference on Hybrid Intelligent Systems (HIS 2018) Held in Porto, Portugal, December 13-15, 2018

粤 Springer 
Editors

Ana Maria Madureira

Ajith Abraham

School of Engineering

Machine Intelligence Research Labs

Instituto Superior de Engenharia (ISEP/IPP)

(MIR Labs)

Porto, Portugal

Auburn, WA, USA

Niketa Gandhi

Machine Intelligence Research Labs

Maria Leonilde Varela

Auburn, WA, USA

Department of Production and Systems

University of Minho

Guimarães, Portugal

ISSN 2194-5357

ISSN 2194-5365 (electronic)

Advances in Intelligent Systems and Computing

ISBN 978-3-030-14346-6

ISBN 978-3-030-14347-3 (eBook)

https://doi.org/10.1007/978-3-030-14347-3

Library of Congress Control Number: 2019933215

(C) Springer Nature Switzerland AG 2020

This work is subject to copyright. All rights are reserved by the Publisher, whether the whole or part of the material is concerned, specifically the rights of translation, reprinting, reuse of illustrations, recitation, broadcasting, reproduction on microfilms or in any other physical way, and transmission or information storage and retrieval, electronic adaptation, computer software, or by similar or dissimilar methodology now known or hereafter developed.

The use of general descriptive names, registered names, trademarks, service marks, etc. in this publication does not imply, even in the absence of a specific statement, that such names are exempt from the relevant protective laws and regulations and therefore free for general use.

The publisher, the authors and the editors are safe to assume that the advice and information in this book are believed to be true and accurate at the date of publication. Neither the publisher nor the authors or the editors give a warranty, express or implied, with respect to the material contained herein or for any errors or omissions that may have been made. The publisher remains neutral with regard to jurisdictional claims in published maps and institutional affiliations.

This Springer imprint is published by the registered company Springer Nature Switzerland AG

The registered company address is: Gewerbestrasse 11, 6330 Cham, Switzerland 


\section{Preface}

Welcome to Porto, Portugal, and to the 18th International Conference on Hybrid Intelligent Systems (HIS 2018); the 10th International Conference on Soft Computing and Pattern Recognition (SoCPaR 2018); and the 13th International Conference on Information Assurance and Security (IAS 2018) held at Instituto Superior de Engenharia do Porto (ISEP) during December 13-15, 2018.

Hybridization of intelligent systems is a promising research field of modern artificial/computational intelligence concerned with the development of the next generation of intelligent systems. A fundamental stimulus to the investigations of hybrid intelligent systems (HISs) is the awareness in the academic communities that combined approaches will be necessary if the remaining tough problems in computational intelligence are to be solved. Recently, hybrid intelligent systems are getting popular due to their capabilities in handling several real-world complexities involving imprecision, uncertainty, and vagueness. HIS 2018 builds on the success of HIS 2017, which was held in Delhi, India, during December 14-16, 2017. HIS 2018 received submissions from 30 countries, and each paper was reviewed by at least five reviewers in a standard peer review process. Based on the recommendation by five independent referees, finally 56 papers were accepted for the conference (acceptance rate of $40 \%$ ).

Conference proceedings are published by Springer Verlag, Advances in Intelligent Systems and Computing Series. Many people have collaborated and worked hard to produce this year successful HIS 2018 conference. First and foremost, we would like to thank all the authors for submitting their papers to the conference and for their presentations and discussions during the conference. Our thanks to Program Committee members and reviewers, who carried out the most difficult work by carefully evaluating the submitted papers. We are grateful to our three plenary speakers:

* Petia Georgieva, University of Aveiro, Portugal

* J. A. Tenreiro Machado, Polytechnic of Porto, Portugal

* Henrique M. Dinis Santos, University of Minho, Portugal 
Our special thanks to the Springer Publication team for the wonderful support for the publication of this proceedings. Enjoy reading!

Ana Maria Madureira Ajith Abraham General Chairs

Maria Leonilde Varela

Oscar Castillo Simone Ludwig Program Chairs 


\section{Contents}

A Machine Learning Approach to Contact Databases' Importation

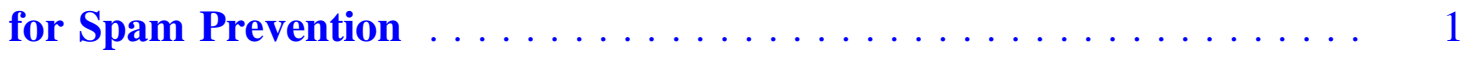
Duarte Coelho, Ana Madureira, Ivo Pereira, and Bruno Cunha

Post-processing of Wind-Speed Forecasts Using the Extended Perfect Prog Method with Polynomial Neural Networks

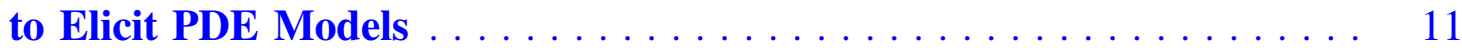
Ladislav Zjavka, Stanislav Mišák, and Lukáš Prokop

Classifying and Grouping Narratives with Convolutional Neural

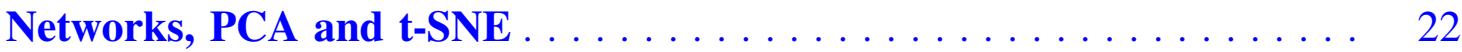
Manoela Kohler, Leonardo Sondermann, Leonardo Forero, and Marco Aurelio Pacheco

Hybrid Instrumental Means of Predictive Analysis of the Dynamics of Natural and Economic Processes

Elena Popova, Luís de Sousa Costa, and Alfira Kumratova

Optimizing Routes for Medicine Distribution Using Team Ant Colony System . .

Renan Costa Alencar, Clodomir J. Santana Jr., and Carmelo J. A. Bastos-Filho

Extending Flow Graphs for Handling

Continuous-Valued Attributes

Emilio Carlos Rodrigues and Maria do Carmo Nicoletti

Modelling and Predicting Individual Salaries in United Kingdom with Graph Convolutional Network

Long Chen, Yeran Sun, and Piyushimita Thakuriah

Predicting the Degree of Collaboration of Researchers

on Co-authorship Social Networks . . . . . . . . . . . . . . . .

Doaa Hassan 


\title{
Hybrid Instrumental Means of Predictive Analysis of the Dynamics of Natural and Economic Processes
}

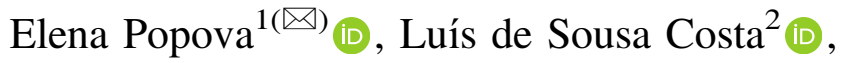 \\ and Alfira Kumratova ${ }^{1}$ \\ 1 Kuban State Agrarian University, \\ 13 Kalinina Str., 350044 Krasnodar, Russian Federation \\ \{elena-popov, alfa05\}@yandex.ru \\ 2 CIMO - Centro de Investigação de Montanha, \\ Departamento Ambiente e Recursos Naturais, Polytechnic Institute of Bragança, \\ Campus de Santa Apolónia, 5300-302 Bragança, Portugal \\ lcosta@ipb.pt
}

\begin{abstract}
The purpose of the presented research is the development and adaptation of mathematical and instrumental methods of analysis and risk management through the forecasting of both economic and natural time series with memory based on the application of new mathematical methods of investigation. The paper poses the problem of developing a constructive method for predictive analysis of time series in the framework of the currently emerging trend of using so-called "graphical tests" in the process of time series' modeling using nonlinear dynamics methods. The main purpose of using graphical tests is to identify both stable and unstable quasiperiodic cycles (quasi-cycles), the whole set of which includes a strange attractor (if one exists). New computer technologies that made it possible to study complex phenomena and processes "on a display screen" were used as instrumentation for the implementation of methods of non-linear dynamics. The proposed approach differs from classical methods of forecasting by new implementation of accounting trends (the evolution of centers and sizes of dimensional rectangles), and appears to be a new tool for identifying cyclic components of the time series in question. As a result, the person, that is making decision has more detailed information, which is impossible to obtain by the methods of classical statistics. The work was supported by Russian Foundation for Basic Research (Grants № 17-06-00354 A).
\end{abstract}

Keywords: Effluents volumes $\cdot$ Harvest $\cdot$ Prediction $\cdot$ Time series · Phase portrait $\cdot$ Bounding box $\cdot$ Quasicycles $\cdot$ Phase analysis

\section{Introduction}

The relevance of the presented study, that is based on the application of the methods of phase analysis to the problems of economic and mathematical modeling of the agroindustrial complex and natural risk factors, is beyond doubt. The authors used in the complex known methods of classical statistics [1-4] and methods of nonlinear dynamics. 
The transition to a new economy calls for the development of new software tools for economic and mathematical modeling, including instruments for risk assessment (prediction and pre-forecast analysis), in particular, such as phase analysis, fractal analysis, linear cellular automaton and dynamic chaos methods.

Concerning the subject of the study - the time series "flow volumes of the Kuban mountain river ", we note the following: in conclusion on the investigation of the flood cause in June 2002 it is said that as a result of rainfall falling in the mountainous areas of the Kuban River basin (with a catchment area of $57,900 \mathrm{~km}^{2}$ the total length of the rivers of the basin is $38325 \mathrm{~km}$, and the total number of rivers is equal to 13569 , the length of the Kuban is $870 \mathrm{~km}$. [5]), there was a flood, which has no analog for almost a hundred-year period of observations, both in terms of maximum flow and rise equal, and for damage caused to the population and enterprises. As a result, 246 settlements were damaged in the South of Russia, more than $110 \mathrm{~km}$ of the gas pipeline, 269 bridges, $1,490 \mathrm{~km}$ of roads, 102 people were killed. The total number of victims in the Southern Federal District reached 340 thousand people, and material damage exceeded 15 billion rubles. Data on the flow volumes of the Kuban River are provided by the Karachaevo-Cherkess Center of Hydrometeorology and Environmental Monitoring - a branch of the Federal State Budget Institution "North Caucasus Department for Hydrometeorology and Environmental Monitoring" of Russia.

Concerning the relevance of the utility of forecasting the values of time series (TS) of crop yields, it can be noted that the importance of planning, achieving and maintaining the development of economic growth rates also in order to ensure a high standard of living for the population, is constantly increasing. It should be noted that planning and forecasting of the enterprise's activities is an objective necessity for any economic system.

\section{Materials and Methods}

The paper presents a comparison of the results of a pre-forecast analysis of time series of a different nature of cyclicity (wheat prices and runoff volumes of the mountain river Kuban) obtained on the basis of phase analysis. We'll demonstrate the method of phase analysis based on the TS of the monthly flow volumes of the mountain river Kuban [5] for the period from 1926 to 2003 (further TS "Kuban"), which has a clear annual cycle [6]. As a comparison, we'll consider the price of wheat per bushel in the American cents from January 1993 to December 2014 (further referred as TS "Wheat"). It should be noted, that a bushel is a unit of volume used in English system of measures. It is mainly used for measuring the volume of agricultural products. One bushel of wheat approximately equals to $27.2 \mathrm{~kg}$.

Let's denote TS "Kuban" as follows:

$$
z=\left\langle z_{i}\right\rangle, i=1,2, \ldots, \mathrm{N}
$$

where $N-$ is the number of TS levels.

In the investigation of this TS, it is sufficiently expedient and informative to construct phase portraits of TS (1) in the phase space $F(Z)[6,7]$ of dimension 2: 
$F(z)=\left\{\left(Z_{i}, Z_{i+1}\right)\right\}, i=1,2, \ldots n-1$. Figure 1 shows the phase trajectory of the TS "Kuban".

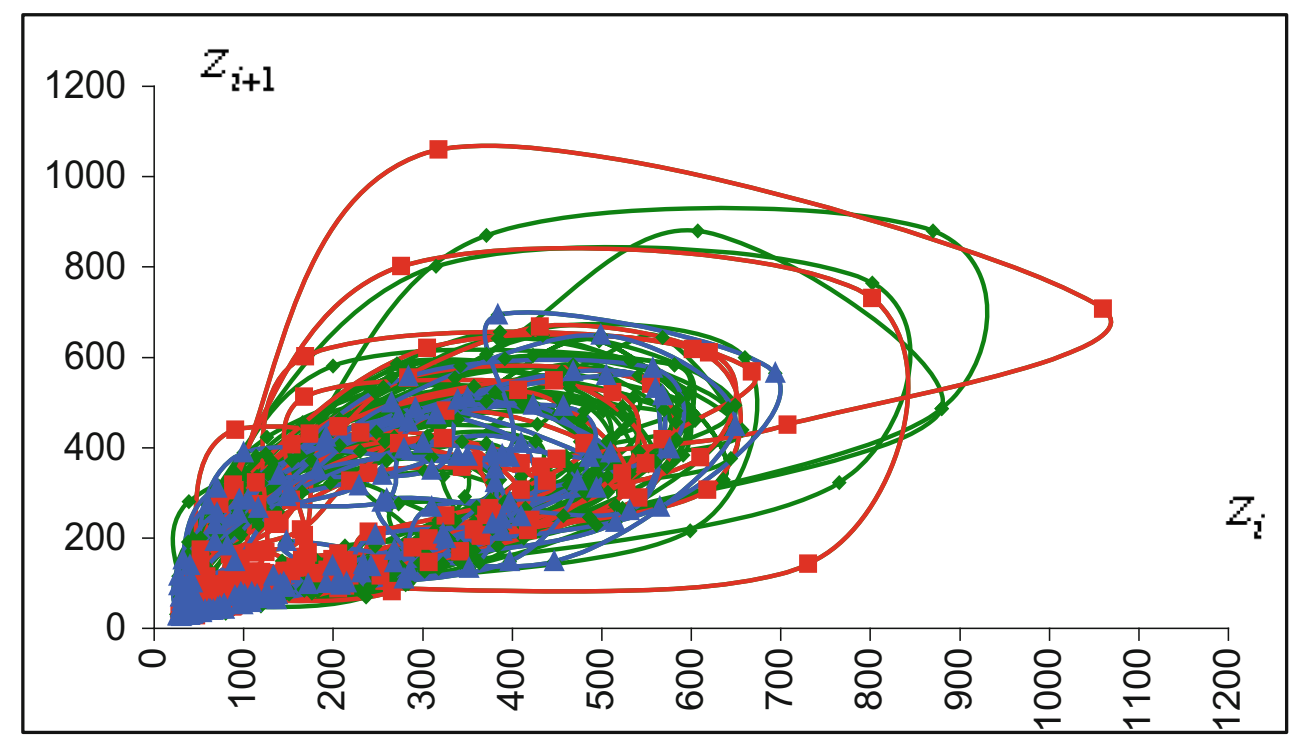

Fig. 1. Phase trajectory of the TS "Kuban"

Following Packard [7], Peters [8] for TS (1) for its phase space we use the formula:

$$
\Psi_{\rho}(Z)=\left\{Z_{i}, Z_{i+1}, \ldots, Z_{n-\rho+1}\right\}, i=1,2, \ldots, n-\rho+1 .
$$

The question of TS dimension $\rho$ is a fundamentally important in the construction of the phase space (2) for a particular TS. This dimension must be no less than the dimension of the attractor of the observed series. As the dimension of the attractor, it can be used the fractal dimension $\mathrm{C}$ of this series. The value of this dimension, as noted in [8], is determined by the following formula:

$$
\mathrm{D}=2-\mathrm{H}
$$

Since the value of the Hurst exponent of the TS's is in the vicinity of $(0 ; 1)$, we can obtain the estimate $\mathrm{D}<2$ [9-11], respectively. From this we can conclude that it is sufficient to use a phase space of dimension for this investigation $\rho=2$.

The definition of the concept of "quasicycle" is described in detail in $[7,11]$.

\section{Results}

\subsection{Approbation of the "Phase Analysis" Program on Real Time Series}

An important and noteworthy feature of TS "Kuban" prediction is that the phase portrait consists of a continuous sequence of disjoint quasicycles whose dimension is equal to a year (exactly 12 months). In general, the trajectory of the phase portrait for 
TS "Kuban" consists of disjoint quasicycles, $\mathrm{C}_{\mathrm{r}}, \mathrm{r}=1,2, \ldots, 16$. Quasicycles are built from February to January, thus forming 12-monthly cycles.

Most of classical research methods have become available due to statistical application packages. The computer began to perform all the laborious, routine and volumetric work on the calculation of various statistical indicators, construction of diagrams and graphs. The researcher mostly performs creative work: setting of the task, determining the method for its solving, obtaining and analyzing the results of the research. Automation of this features is the goal that is pursued by the program "Phase Analysis" $(\mathrm{C}++)$, developed and presented by the authors in this paper; the program can be used for automatic realization of phase analysis calculations (in accordance with Fig. 4). The program has a convenient interface and enables the economist-expert to implement the analytical process.

In terms of the phase analysis' tools, a separate annual cycle belonging to TS Z (1) is presented as a typical quasicycle inherent to TS "Kuban" (Fig. 2(b)).

Along with TS "Kuban" (in accordance with Fig. 3(a)) phase portraits for TS "Wheat" (in accordance with Fig. 3(b)) were constructed.

The latter is due to the existence of the lag in the work of the sequential analysis algorithm. In this case, the size of lag is 3 . The mentioned above lag is represented by three points 13, 14 and 15 (in accordance with Fig. 2(a)).

The definition of the term "quasicycle" is in a sense close to the definition of the generally accepted concept of a "cycle". The difference between these two concepts is that the initial and final points of the quasicycle do not necessarily have to coincide. The end point of a quasicycle is determined by its occurrence in a neighborhood of the initial point. In this case, self-intersection of the initial and final links of the quasicycle is allowed, if this leads to the best approximation of its initial and final points. Figure 3 shows examples of quasicycles that are obtained after the decomposition of the phase portrait in Fig. 1 into quasicycles. Table 1 shows the dimensions $L_{k}$ of all 15 quasicycles.

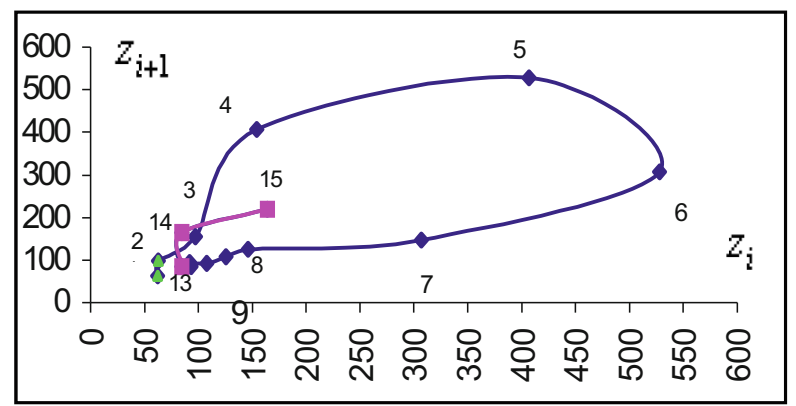

(a)

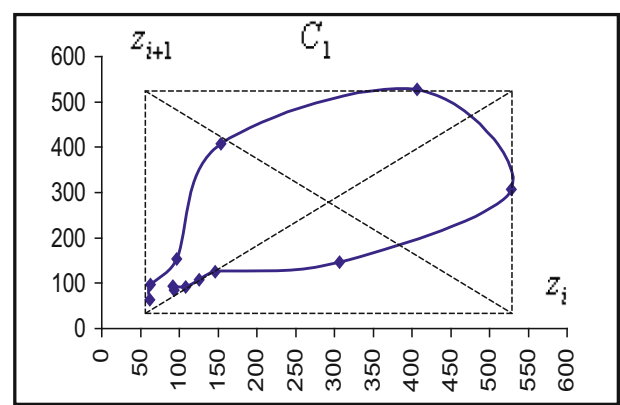

(b)

Fig. 2. (a) The first quasicycle of the phase portrait $\Psi_{2}(Z)$, including the lag - 13, 14, 15; (b) The first quasicycle TS Z, whose size is 12 


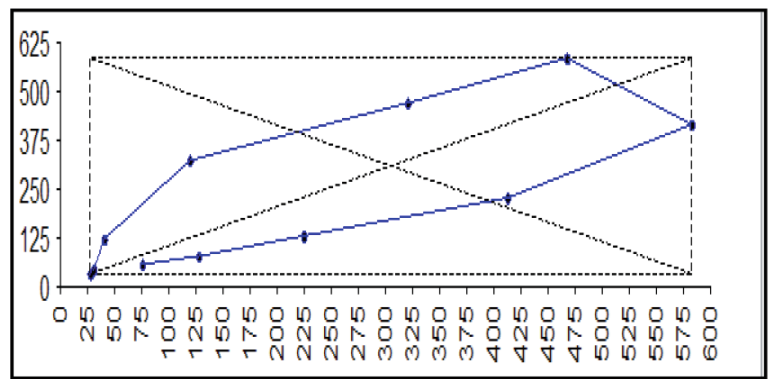

(a)

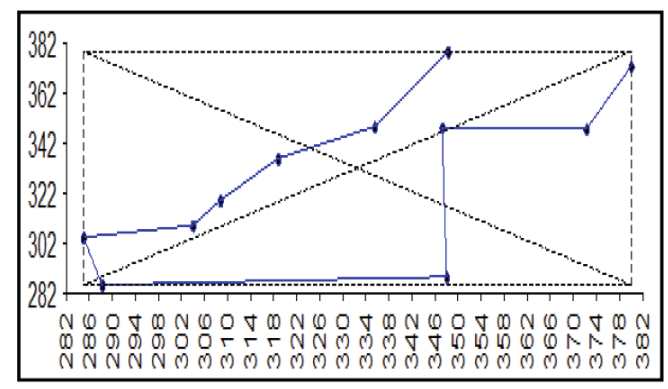

(b)

Fig. 3. The "Phase Portraits" tab of the "Phase Analysis" program with automatic construction of dimensional rectangles (represented by dotted lines): (a) TS "Kuban"; (b) TS "Wheat".

Table 1. The dimensions of all quasicycles

\begin{tabular}{l|l|l|l|l|l|l|l|l|l|l|l|l|l|l|l}
\hline № $C_{k}$ & 1 & 2 & 3 & 4 & 5 & 6 & 7 & 8 & 9 & 10 & 11 & 12 & 13 & 14 & 15 \\
\hline$L_{k}^{r}$ & 12 & 12 & 12 & 12 & 12 & 12 & 12 & 12 & 12 & 12 & 12 & 12 & 12 & 12 & 12 \\
\hline
\end{tabular}

We denote $\mathrm{Z}_{\mathrm{k}}$ by this segment $\mathrm{BP} \mathrm{Z}$, which is obtained by the removal procedure from all observation $Z$ points related to quasicycles $C_{1}, C_{2}, \ldots, C_{k-1}$; according to this definition $\mathrm{Z}_{1}=\mathrm{Z}$.

From Table 1 we can make a conclusion that the presence of long-term memory in the considered TS, along with other factors, is due to the cyclic component of this TS.

For each quasicycle $C_{k}$, a "overall rectangle of a quasicycle $C_{k}$ " is constructed [10]. The intersection of the diagonals of the overall rectangle defines the so-called "center of rotation" of the quasicycle $\mathrm{O}_{\mathrm{k}}$, whose coordinates are denoted by $\mathrm{O}_{\mathrm{k}}\left(\mathrm{x}_{\mathrm{k}}, \mathrm{y}_{\mathrm{k}}\right)$. The construction of such a rectangle consists of the following operations. First, in the considered quasicycles $\mathrm{K}_{\mathrm{r}}^{1}$ two points are distinguished: the first with the minimum abscissa value, the second with the maximum abscissa value through these selected points we draw dashed lines which are parallel to the axis of ordinates. Further, in the quasicycle $\mathrm{C}_{1}$ two points are distinguished: the first - with the minimum value of the ordinate, the second - with the maximum value of the ordinate; through these selected points we draw with dashed lines segments, parallel to the axis of abscissae. The intersection of the constructed two pairs of parallel lines forms the required dimensional rectangle for the quasicycle in question $\mathrm{C}_{1}$; the center of this quasicycle is represented by the point of intersection of the diagonals of the overall rectangle (example is shown on Fig. 3).

Step-by-step algorithm for phase analysis

Based on the analysis carried out by the authors, the following approach for predicting the TS of the considered type is proposed; it consists of the following steps:

1. Conducting a fractal analysis of TS (1) in order to establish the presence of a longterm memory to evaluate its depth $\rho$. Wherein we get a fuzzy set of estimates $L=L(Z)=\left\{\left(l, \mu_{l}\right)\right\}$ of the depth of the memory of TS $z$.

2. The construction of a phase portrait for the indicated TS. 
3. Decomposition of the phase portrait into quasicycles $C_{r}$.

4. Analysis of the movement of the centers of quasicycles $O_{r}\left(x_{r}, y_{r}\right)$, the movement of the dimensions of the overall rectangles' of quasicycles areas, and also the directions of rotation of the links of quasicycles.

5. Construction of the forecast by the principle of continuation (completion) of the corresponding quasicycle using the results of stage 4 for two cases, when the last quasicyclic is:

(a) incomplete (we use the overall dimensions and the pattern of the rotation of quasicycles, considering the sector of the overall rectangle to which the predicted point belongs);

(b) completed (we use the overall dimensions and the pattern of the rotation of quasicycles, considering the evolution of the centers and the transitions from the final point of one cycle to the starting point of a new cycle).

\section{Discussion}

Let's list the revealed features of the phase trajectories of the TS "Kuban".

1. The phase portrait of the TS "Kuban" consists of quasicycles with a dimension that equals 12. This fact does not contradict the results of a fractal analysis on the evaluation of the depth of the memory of TS [6].

2. It was considered that each link of all quasicycles rotates clockwise. The overall rectangle can be divided into 4 sectors by straight lines.

3. The centers of quasicycles $O_{k}\left(x_{k}, y_{k}\right)$, in the order of their numbering $k=\overline{1.72}$, evolve along a definite trajectory, whose points are located in a sufficiently small vicinity of the bisector of the positive orthant of Cartesian coordinates.

The coordinates of the centers of all quasicycles define the points of the bisector of the positive orthant $x_{k} x_{k+1}$ of the Cartesian coordinates (in accordance with Fig. 4). Note that the trajectory of the motion of these coordinates is characterized by an wide range $K \approx 550-200=350$, which exceeds the minimum point by more than 1.5 times.

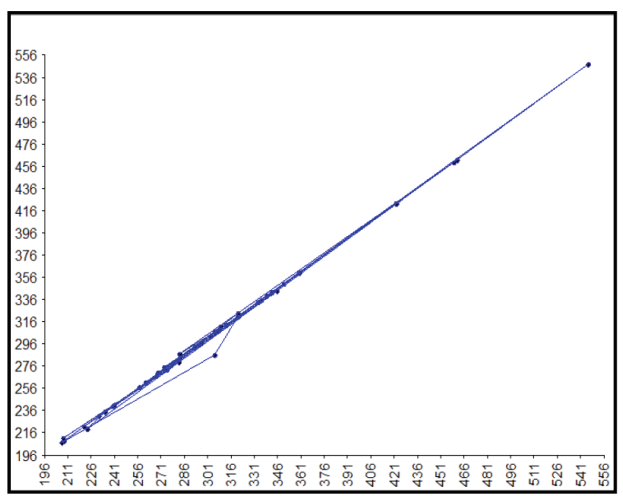

(a)

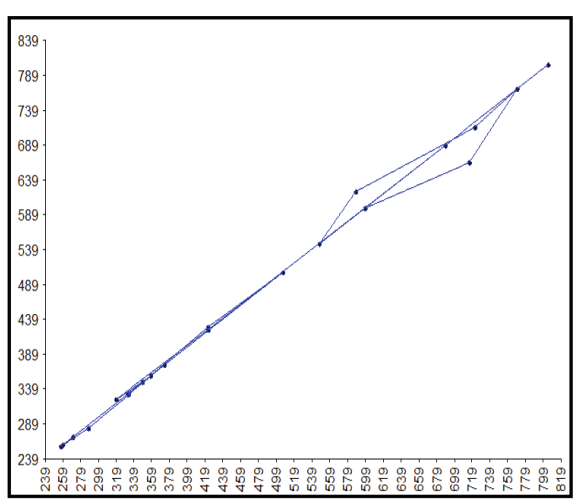

(b)

Fig. 4. Motion of the centers of quasicycles (the values of the coordinate axes for TS "Kuban" are represented by the values of river flow volumes (thousand cubic meters), for "Wheat" - by the price for bushel in US cents): (a) TS "Kuban"; (b) TS "Wheat". 
In connection with the above mentioned, it is practical interesting to determine the long-term trends that govern the motion of the centers of the dimensional rectangles. For this purpose, the division for the periods was performed (Fig. 5): from 1926 to 1940 (in accordance with Fig. 5a); from 1946 to 1987 (in accordance with Fig. 5b); from 1988 to 2003 (in accordance with Fig. 5c). Visualization of Fig. 4 reveals the following trend: at approximately the same value $\min \approx 200$, in the process of time the value of the range increases in the following ratio:

$\mathrm{R}_{1} \approx 350-200=150$ (in accordance with Fig. 5a)

$\mathrm{R}_{2} \approx 450-200=200$, (in accordance with Fig. 5b)

$\mathrm{R}_{3} \approx 550-200=350$ (in accordance with Fig. 5c). A well-known climatologists' statement says about the existence of a general trend of climate warming in the northern hemisphere confirms the above-mentioned range of magnitude values as filling of mountain rivers is determined by the intensity of the glaciers' melting, especially in summer months.

4. The movement of the dimensions (area) of the overall rectangles of quasicycles is cyclical, which is confirmed by the visualization of Fig. 6.

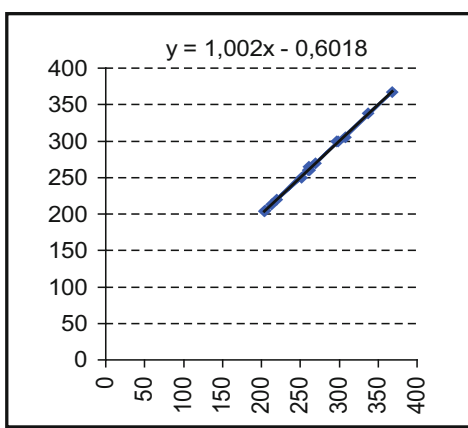

(a)

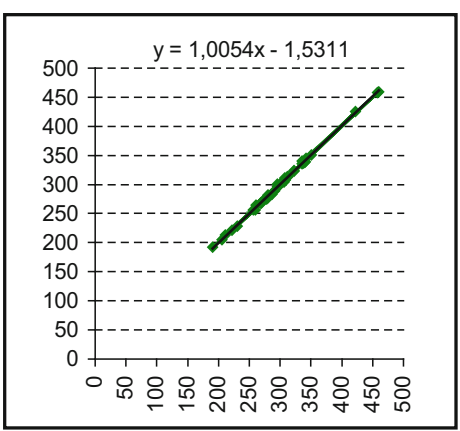

(b)

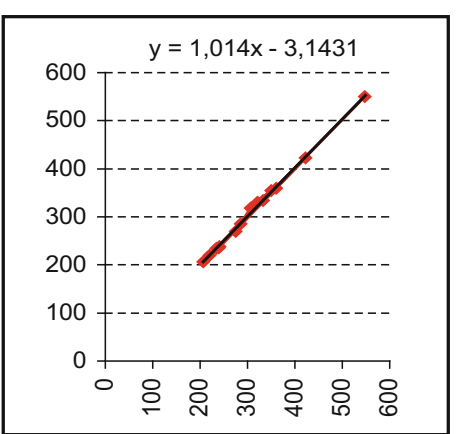

(c)

Fig. 5. Evolution of the area of the overall rectangles, considering time parameter

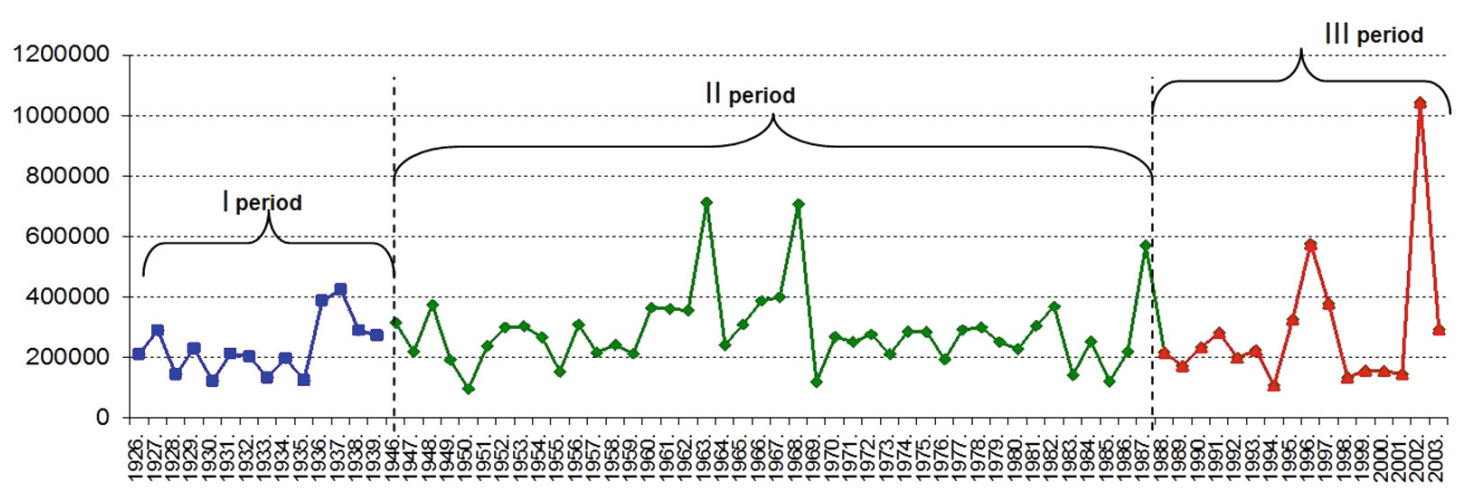

Fig. 6. Movement of areas of dimensional rectangles (taking into account time parameter) 
It is important to mention that authors carried out graphical testing of time series, both natural, economic, and financial. The fact of confirming a 12-month cycle in natural and economic series with a pronounced annual seasonal cyclicity based on phase analysis allowed to use it for other time series. For example, financial monthly TS of pairs euro/ruble, dollar/ruble, gold, silver, palladium, platinum have quasi-cycles of various lengths from 3 to 8 months (Table 2). From the obtained results of phase analysis, it is reasonable to expect a sufficiently high degree of reliability in predicting general trends for TS elements of the financial market using models based on the use of long-term memory, in particular using a cellular automaton predictive model [1].

Table 2. Results of phase analysis (PhA) for the elements of the financial market time series

\begin{tabular}{l|l|l}
\hline $\begin{array}{l}\text { Financial market } \\
\text { element }\end{array}$ & $\begin{array}{l}\text { Mean length of quasicycles } \\
(\mathrm{PhA})\end{array}$ & $\begin{array}{l}\text { Quasi-cycles lengths' depth of } \\
\text { memory (PhA) }\end{array}$ \\
\hline Euro/ruble & 5.83 & $5-6$ \\
\hline Dollar/ruble & 7.2 & $7-8$ \\
\hline Gold & 6.22 & $7-8$ \\
\hline Silver & 6.81 & $\mathbf{5 - 6}$ \\
\hline Palladium & 6 & $6-7$ \\
\hline Platinum & 6.58 & $6-7$ \\
\hline
\end{tabular}

In this way, the proposed approach differs from the classical methods of forecasting by the new trend's registration implementation (evolution of the centers and sizes of the overall rectangles), represents a new tool (phase portraits) for identifying the cyclic component of the TS, which in its turn makes it possible to identify the prognostic properties of the series under study [2] and thus identify ways to reduce socioeconomic risks.

\section{References}

1. Adamowski, J., Chan, H.F., Prasher, S.O., Sharda, V.N.: Comparison of multivariate adaptive regression splines with coupled wavelet transform artificial neural networks for runoff forecasting in Himalayan micro-watersheds with limited data. J. Hydroinf. 14, 731744 (2012). https://doi.org/10.2166/hydro.2011.044

2. Yaseen, Z.M., Kisi, O., Demir, V.: Water Resour. Manag. 30, 4125 (2016). https://doi.org/ 10.1007/s11269-016-1408-5

3. Ahani, A., Shourian, M., Rahimi Rad, P.: Water Resour. Manag. 32, 383 (2018). https://doi. org/10.1007/s11269-017-1792-5

4. Parmar, K.S., Bhardwaj, R.: Appl. Water Sci. 4, 425 (2014). https://doi.org/10.1007/s13201014-0159-9

5. Popular science encyclopedia "Water of Russia". http://water-rf.ru. Accessed 11 July 2018

6. Vintizenko, I.G.: Deterministic forecasting in economic systems. New technologies in management, business and law. Nevinnomyssk, pp. 163-167 (2003)

7. Packard, N., Crutchfield, J., Farmer, D., Shaw, R.: Geometry from a time series. Phys. Rev. Lett. 45, 712 (1980) 
8. Peters, E.: Chaos and Order in the Capital Markets. A New Analytical View of Cycles, Prices and Market Volatility, p. 333. Wiley, Hoboken (2000)

9. Kumratova, A.M.: The study of trend-seasonal processes using classical statistics. Polythematic Netw. Electron. Sci. J. KubSAU, Krasnodar, Russia 103(09), 312-323 (2014)

10. Kumratova, A.M., Popova, E.V., Kurnosova, N.S.: Reduction of economic risk based on pre-forecast analysis. In: Modern Economy: Problems and Solutions, Voronezh, Russia, pp. 18-27 (2015)

11. Kumratova, A.M., Popova, E.V.: Evaluation and risk management: analysis of time series by methods of nonlinear dynamics, 212 p. KubSAU, Krasnodar, Russia (2014) 